\title{
Combination of Baseline LDH, Performance Status and Age as Integrated Algorithm to Identify Solid Tumor Patients with Higher Probability of Response to Anti PD-1 and PD-L1 Monoclonal Antibodies
}

\author{
Maria Silvia Cona ${ }^{1}$, Mara Lecchi ${ }^{2}$, Sara Cresta ${ }^{1}$, Silvia Damian ${ }^{1}$, Michele Del Vecchio ${ }^{1}$, \\ Andrea Necchi ${ }^{1}$, Marta Maria Poggi ${ }^{1}$, Daniele Raggi ${ }^{1}$, Giovanni Randon ${ }^{1}$, Raffaele Ratta ${ }^{1}$, \\ Diego Signorelli ${ }^{1}$, Claudio Vernieri ${ }^{1,3}$, Filippo de Braud ${ }^{1,4}$, Paolo Verderio ${ }^{2}$ and \\ Massimo Di Nicola ${ }^{1, *}$ \\ 1 Medical Oncology Unit, Fondazione IRCCS, Istituto Nazionale dei Tumori di Milano, \\ Via Giacomo Venezian 1, 20133 Milan, Italy; mariasilvia.cona@istitutotumori.mi.it (M.S.C.); \\ sara.cresta@istitutotumori.mi.it (S.C.); silvia.damian@istitutotumori.mi.it (S.D.); \\ michele.delvecchio@istitutotumori.mi.it (M.D.V.); andrea.necchi@istitutotumori.mi.it (A.N.); \\ martamariapoggi@gmail.com (M.M.P.); daniele.raggi@istitutotumori.mi.it (D.R.); \\ giovanni.randon@istitutotumori.mi.it (G.R.); raffaele.ratta@istitutotumori.mi.it (R.R.); \\ diego.signorelli@istitutotumori.mi.it (D.S.); claudio.vernieri@istitutotumori.mi.it (C.V.); \\ filippo.debraud@istitutotumori.mi.it (F.d.B.) \\ 2 Bioinformatics and Biostatistics Unit, Fondazione IRCCS, Istituto Nazionale dei Tumori di Milano, \\ Via Giacomo Venezian 1, 20133 Milan, Italy; mara.lecchi@istitutotumori.mi.it (M.L.); \\ paolo.verderio@istitutotumori.mi.it (P.V.) \\ 3 IFOM (Fondazione Istituto FIRC di Oncologia Molecolare), via Adamello 16, 20139 Milan, Italy \\ 4 Department of Oncology and Hemato-Oncology, Universita' degli Studi di Milano, 20122 Milan, Italy \\ * Correspondence: massimo.dinicola@istitutotumori.mi.it
}

Received: 12 December 2018; Accepted: 12 February 2019; Published: 14 February 2019

check for updates

\begin{abstract}
Predictive biomarkers of response to immune-checkpoint inhibitors (ICIs) are an urgent clinical need. The aim of this study is to identify manageable parameters to use in clinical practice to select patients with higher probability of response to ICIs. Two-hundred-and-seventy-one consecutive metastatic solid tumor patients, treated from 2013 until 2017 with anti- Programmed death-ligand 1 (PD-L1)/programmed cell death protein 1 (PD-1) ICIs, were evaluated for baseline lactate dehydrogenase (LDH) serum level, performance status (PS), age, neutrophil-lymphocyte ratio, type of immunotherapy, number of metastatic sites, histology, and sex. A training and validation set were used to build and test models, respectively. The variables' effects were assessed through odds ratio estimates (OR) and area under the receive operating characteristic curves (AUC), from univariate and multivariate logistic regression models. A final multivariate model with LDH, age and PS showed significant ORs and an AUC of 0.771. Results were statistically validated and used to devise an Excel algorithm to calculate the patient's response probabilities. We implemented an interactive Excel algorithm based on three variables (baseline LDH serum level, age and PS) which is able to provide a higher performance in response prediction to ICIs compared with LDH alone. This tool could be used in a real-life setting to identify ICIs in responding patients.
\end{abstract}

Keywords: immune-checkpoint inhibitors; LDH; biomarkers 


\section{Introduction}

In the era of immunotherapy, several biological and biochemical factors have been investigated as potential biomarkers of tumor response/resistance to immune-checkpoint inhibitors (ICIs). Select patients is an important clinical need in an attempt to offer them the best therapeutic workup, to avoid unnecessary side effects, and to optimize the use of economic resources. In order to identify a predictive tool of response to ICIs, we evaluated the available and manageable parameters that could ameliorate the selection of patients. In this context, lactate dehydrogenase (LDH) is a potentially interesting, cheap and easy-to-detect biomarker of response [1-4]. Indeed, serum LDH levels are an independent poor prognostic factor in several malignancies, including renal cell [5] and nasopharyngeal carcinoma [6], lymphomas [7], multiple myeloma [8], sarcomas [9] and lung cancer $[10,11]$. It also seems to be predictive of clinical outcomes in patients treated with anti-PD1 monoclonal antibodies (mAbs) [12]. For instance, it inversely correlates with the probability of achieving a tumor response in metastatic melanoma patients treated with anti-Cytotoxic T-Lymphocyte Antigen 4 (CTLA4) mAbs [13,14]. In previous studies, the link between serum LDH levels and poor patient prognosis has been generally attributed to the fact that high LDH levels reflect a high tumor burden, which is often associated with worse clinical outcomes. However, LDH is an enzyme that catalyses the conversion of pyruvate to lactate in highly glycolytic cancer cells, and its serum levels could be a proxy of tumor metabolic activity and not simply of tumor burden. Of note, recent studies have suggested that enhanced glycolytic activity in human malignancies is associated with an immunosuppressive environment, while glycolysis inhibition reduces tumor infiltration by immunosuppressor myeloid cells (MDSCs), stimulating the infiltration by cytotoxic lymphocytes [15].

Potential biomarkers of response/resistance to immunotherapy other than serum LDH, such as intratumor Programmed death-ligand 1 (PD-L1) expression, tumor microenvironment characteristics, tumor mutational load, mismatch-repair deficiency, and neutrophil-lymphocyte (N/L) ratio in peripheral blood, have been extensively investigated [16]. Unfortunately, no single parameter has been consistently associated with tumor response and clinical outcomes in all types of neoplasms; moreover, many of these biomarkers require specific analyses in tumor specimens, which are not always available. Therefore, cheap and easy-to-measure biochemical and clinical parameters could significantly help in the selection of patients more likely to benefit from ICIs, without increasing costs.

Here, we describe an algorithm based on baseline serum LDH levels, patient Performance Status (PS) and age, which could help clinicians to provide more accurate identification of patient candidates to ICIs.

\section{Results}

In our study, we enrolled 271 metastatic solid tumor patients treated at Fondazione IRCCS-Istituto Nazionale dei Tumori with anti PD-1 and anti PD-L1 mAbs from April 2013 to August 2017. Patients were evaluated for baseline LDH serum level, PS, age, N/L ratio, type of immunotherapy, number of metastatic sites, histology, and sex. Overall, the population was made up of $43.2 \%$ (117) lung cancer, $22.1 \%$ (60) melanoma and 34.7\% (94) miscellaneous other solid tumors (1 anal, 1 hepatocellular carcinoma (HCC), 1 thyroid, 1 germ cell tumor, 2 gynecologic, 3 gastric, 5 head and neck (H\&N), 4 colorectal, 5 sarcoma, 6 biliary tract, 6 mesothelioma, 26 renal, and 33 urothelial). Patient's characteristics of training, validation and overall cohort are reported in Table 1; the number of metastatic sites is defined as the number of involved organs; the PS is evaluated through the Eastern Cooperative Oncology Group (ECOG) criteria [17] and dichotomized as 0 or $\geq 1$. All responses were assessed by computed tomography. The categories of response consisted of: complete response (CR), partial response (PR), stable disease (SD), or disease progression (DP) as per RECIST (Response Evaluation Criteria in Solid Tumours) 1.1 criteria [18]. Disease control (DC) was defined as any CR, PR or SD. Overall, 150 (55.35\%) patients achieved DC (6 CR, 59 PR, 85 SD) and 121 (44.65\%) had DP as their best response. 
Table 1. Clinic-pathological characteristics: training, validation and total cohorts.

\begin{tabular}{|c|c|c|c|c|c|c|}
\hline \multirow{2}{*}{$\begin{array}{c}\text { Characteristics } \\
\text { Categorical Variables }\end{array}$} & \multicolumn{2}{|c|}{ Training Cohort } & \multicolumn{2}{|c|}{$\begin{array}{c}\text { Validation } \\
\text { Cohort }\end{array}$} & \multicolumn{2}{|c|}{ Total Cohort } \\
\hline & Freq & $\%$ & Freq & $\%$ & Freq & $\%$ \\
\hline \multicolumn{7}{|l|}{ Sex } \\
\hline Female & 78 & 41.71 & 32 & 38.1 & 110 & 40.59 \\
\hline Male & 109 & 58.29 & 52 & 61.9 & 161 & 59.41 \\
\hline \multicolumn{7}{|l|}{ Tumor Site } \\
\hline Melanoma & 36 & 19.25 & 24 & 28.57 & 60 & 22.14 \\
\hline Lung & 93 & 49.73 & 24 & 28.57 & 117 & 43.17 \\
\hline Others * & 58 & 31.02 & 36 & 42.86 & 94 & 34.69 \\
\hline \multicolumn{7}{|l|}{ Treatment } \\
\hline PD-1 & 117 & 62.57 & 51 & 60.71 & 168 & 61.99 \\
\hline PD-L1 & 70 & 37.43 & 33 & 39.29 & 103 & 38.01 \\
\hline \multicolumn{7}{|l|}{ Line of therapy } \\
\hline 1 & 8 & 4.3 & 37 & 44.0 & 45 & 16.61 \\
\hline 2 & 56 & 29.9 & 33 & 39.3 & 89 & 32.84 \\
\hline$\geq 3$ & 123 & 65.8 & 14 & 16.7 & 137 & 50.55 \\
\hline \multicolumn{7}{|l|}{ Number of Metastatic sites } \\
\hline 1 & 31 & 16.6 & 11 & 13.1 & 42 & 15.5 \\
\hline 2 & 83 & 44.4 & 37 & 44.0 & 120 & 44.28 \\
\hline$\geq 3$ & 73 & 39.0 & 36 & 42.9 & 109 & 40.22 \\
\hline \multicolumn{7}{|l|}{ PS (ECOG) } \\
\hline 0 & 123 & 65.78 & 54 & 64.29 & 177 & 65.31 \\
\hline$\geq 1$ & 64 & 34.22 & 30 & 35.71 & 94 & 34.69 \\
\hline \multicolumn{7}{|l|}{ Best Response } \\
\hline DC & 104 & 55.61 & 46 & 54.76 & 150 & 55.35 \\
\hline DP & 83 & 44.39 & 38 & 45.24 & 121 & 44.65 \\
\hline Continuos variables & Median & Range & Median & Range & Median & Range \\
\hline Age, years & 61 & $16 ; 84$ & 66 & $34 ; 83$ & 62 & $16 ; 84$ \\
\hline $\mathrm{N} / \mathrm{L}$ ratio & 3.44 & $\begin{array}{r}0.65 \\
39.50\end{array}$ & 3.39 & $\begin{array}{r}0.78 \\
28.33\end{array}$ & 3.44 & $\begin{array}{r}0.65 \\
39.50\end{array}$ \\
\hline LDH serum level & 353.00 & $\begin{array}{l}152.00 \\
2048.00\end{array}$ & 321.50 & $\begin{array}{c}179.00 \\
5063.00\end{array}$ & 343.00 & $\begin{array}{l}152.00 \\
5063.00\end{array}$ \\
\hline
\end{tabular}

PS: performance status, DC: disease control, DP: disease progression, N/L ratio: neutrophil to lymphocyte ratio. * Other solid tumors: 1 anal, 1 HCC, 1 thyroid, 1 germ cell tumor, 2 gynecologic, 3 gastric, 5 H\&N, 4 colorectal,

5 sarcoma, 6 biliary tract, 6 mesothelioma, 26 renal, and 33 urothelial.

The evaluation of LDH levels was performed in terms of percentage increase with respect to the upper limit of the specific normality range. This transformed variable was called $L D H$ normalized. The median value of $L D H$ normalized distribution was $-27.7 \%$, the lower quartile $-38.48 \%$ and the upper $-3.96 \%$. A minimum extreme value was observed at $-66.96 \%$ and a maximum at $954.79 \%$. Figure 1 shows the $L D H$ normalized distributions in the training and validation cohorts when considering all patients (Figure 1A) or according to the best response achieved with immunotherapy (Figure 1B).

For all the continuous variables considered in the logistic regression model, we found that a linear relationship between the log odds and their values was satisfied. 

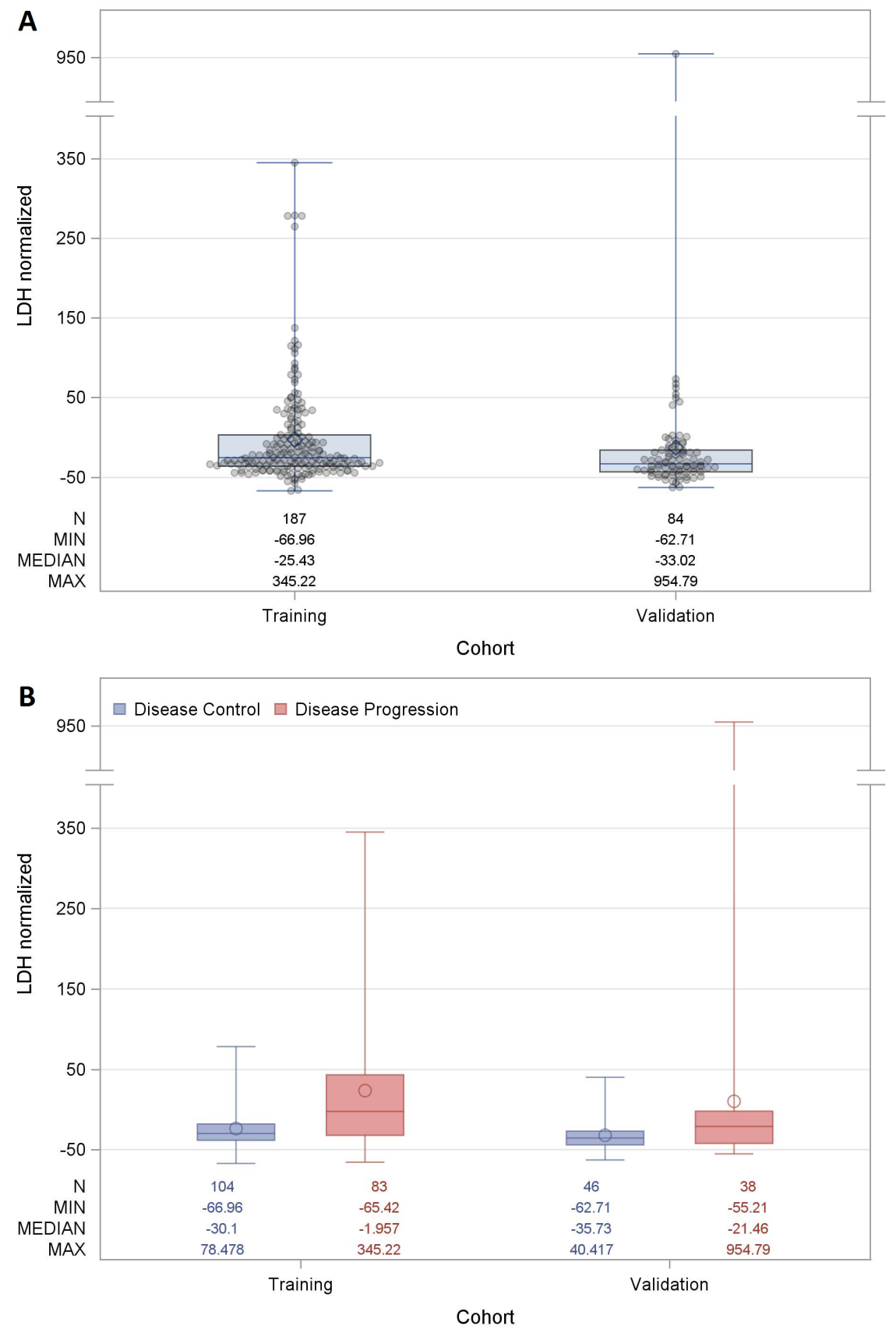

Figure 1. Distribution of lactate dehydrogenase $(L D H)$ normalized in the training and validation cohort. (A) Boxplots reflecting the distribution of $L D H$ normalized for each patient $(n=271)$ distinguished in training and in the validation cohort. Each box indicates the 25th and 75th centiles. The horizontal line inside the box indicates the median, and the whiskers indicate the extreme measured values. Each observation is represented by a grey dot. (B) Boxplots reflecting the distribution of LDH normalized according to the best response distinguished in training and in the validation cohort. Each box indicates the 25th and 75th centiles. Blue and red colors indicate disease control and disease progression patients, respectively. The horizontal line inside the box indicates the median, and the whiskers indicate the extreme measured values.

Univariate analysis was performed in 104 patients achieving DC and 83 patients undergoing DP (training set); of note, clinical response was significantly associated $(p<0.0001)$ with LDH normalized, with an odds ratio estimate (OR) equal to 0.792 for any $\mathrm{LDH}$ increment of $10 \%$. We also found a 
significant positive association between age and tumor response, with an OR of 1.426 for any 10-year increment ( $p$-value: 0.0093), and an inverse association between ECOG PS with an OR equal to 0.530 for 1 vs. 0 score ( $p$-value: 0.0419 ) or N/L ratio with an OR equal to 0.899 ( $p$-value: 0.014 ) and tumor response. Then, a logistic multivariate model was built by including these four variables, and a backward selection procedure was performed. Baseline LDH serum levels, age and PS were independently associated with the probability of responding to the treatment, with a statistically significant $(p<0.05)$ or borderline significant ( $p$-value: 0.056 in the case of PS) association; therefore, they were retained in the final model. On the other hand, the N/L ratio was removed because it was not independently associated with the chance of responding ( $p$-value: 0.529$)$. The predictive capability of the final model was evaluated by generating a receive operating characteristic curve (ROC) and using as a pivotal statistic the area under the ROC curve (AUC). A satisfactory predictive capability [19] was observed, showing an AUC of 0.771 (95\% Confidence Interval (CI): 0.701;0.842). The contribution of each variable of the final model to the predictive performance is graphically shown in Figure 2, and the differences between AUCs of the LDH normalized univariate model and the final one turned out to be significantly different to zero (difference: -0.0585 ; $p$-value: $0.0298 ; 95 \%$ CI: $-0.111 ;-0.0057)$. By applying the training coefficients to the validation set, the model was statistically validated showing a significant AUC of 0.685 (95\% CI: 0.569;0.801) (Figure 3). When the validated model was fitted to the totality of 150 patients achieving DC and 121 patients undergoing DP, the impact of these three variables on tumor response remained significant, as shown in Table 2; the overall AUC value, as well as the cross-validated one, were satisfactory (AUC: 0.737 95\% CI: 0.675;0.798 and AUC: 0.718 95\% CI: 0.654;0.781, respectively). Finally, we implemented an interactive Excel tool like that shown, for feasibility purposes, in the example of Table 3. By inserting for each patient: the upper limit of the normal reference range of the adopted kit for LDH quantification, the baseline LDH serum value, the ECOG PS score (as 0,1,2), and the age, it is possible to obtain the corresponding estimated probability of clinical response to ICIs.

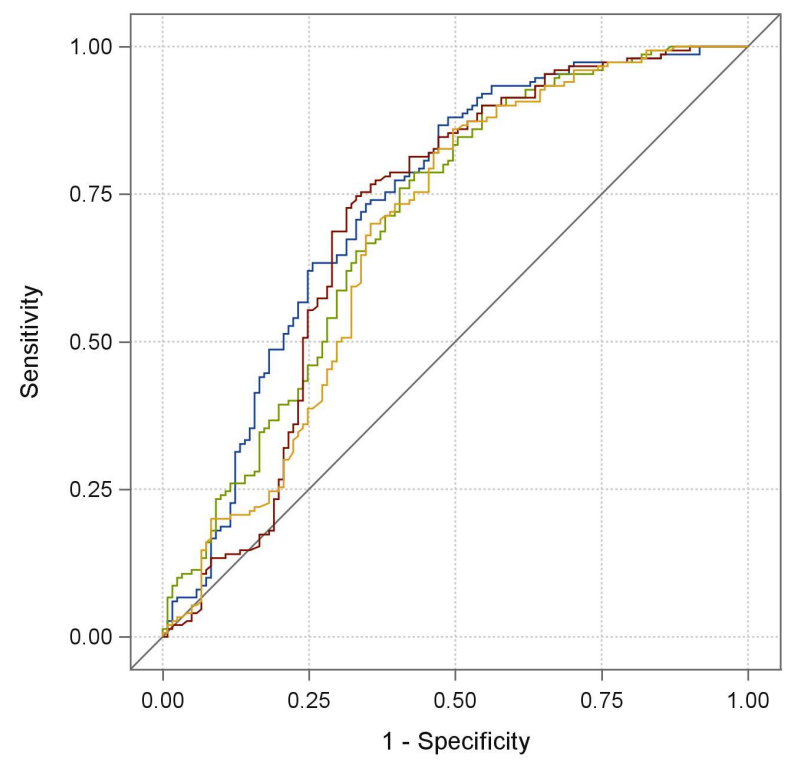

Figure 2. Receive Operating Characteristic (ROC) curves in the training set of the final multivariate model (blue line, Area under the ROC curve (AUC): 0.771), final model without performance status (green line, AUC: 0.749), final model without age (red line, AUC: 0.728), and LDH normalized univariate model (yellow line, AUC: 0.713). 


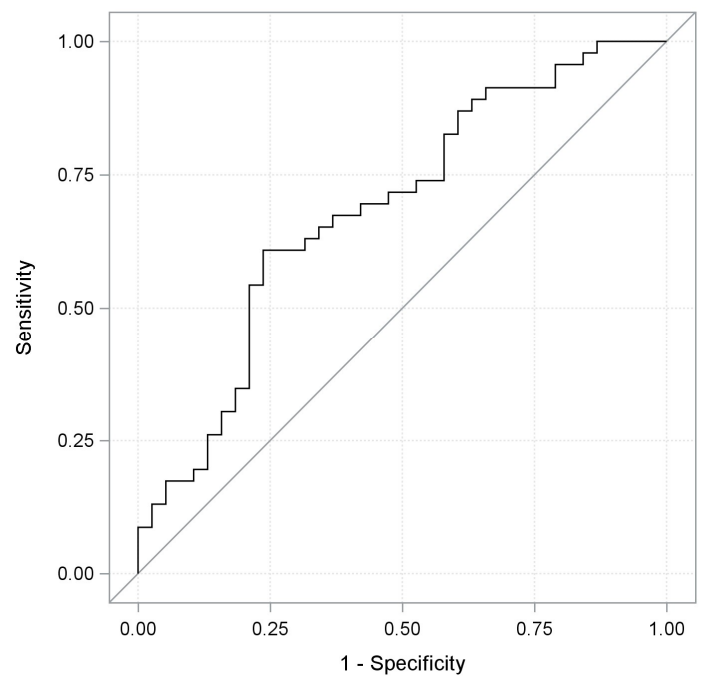

Figure 3. ROC curve of the final multivariate model applied on the validation set with an AUC value of 0.685 .

Table 2. Overall Odd Ratio (OR) estimates and 95\% Confidence Interval (CI) for each variable of the final model.

\begin{tabular}{cccc}
\hline Effect & OR & \multicolumn{2}{c}{ 95\% CI } \\
\hline LDH normalized for a 10\% increment & 0.810 & 0.744 & 0.883 \\
Age for a ten-years increment & 1.305 & 1.038 & 1.641 \\
PS (ECOG) 1 vs. 0 score & 0.481 & 0.274 & 0.846
\end{tabular}

OR: Odd Ratio; CI: Confidence Interval; PS: performance status; ECOG: Eastern Cooperative Oncology Group criteria.

Table 3. Example of the excel interactive tool. Grey cells need to be filled; the blue one will show the estimated probability of clinical response.

\begin{tabular}{cc}
\hline Variable & Value \\
\hline Kit Characteristic & \\
Upper limit of normal reference range & 460 \\
Patients Characteristics & \\
LDH serum value & 77 \\
ECOG PS score [17] & 1 \\
Age & 60 \\
Estimated Probability \% & 76.39 \\
\hline
\end{tabular}

Finally, we compared the performance (in terms of AUC) of the predictor built starting from the final model, to that derived from the only N/L ratio. As reported in Figure 4, the first classifier, with an AUC equal to 0.737 (95\% CI: 0.675; 0.798), showed a higher predictive capability with respect to the N/L ratio classifier characterized by an AUC value of 0.645 (95\% CI: 0.579; 0.711). In particular, the AUC values' difference was statistically significant ( $p$-value: 0.0220 ). 


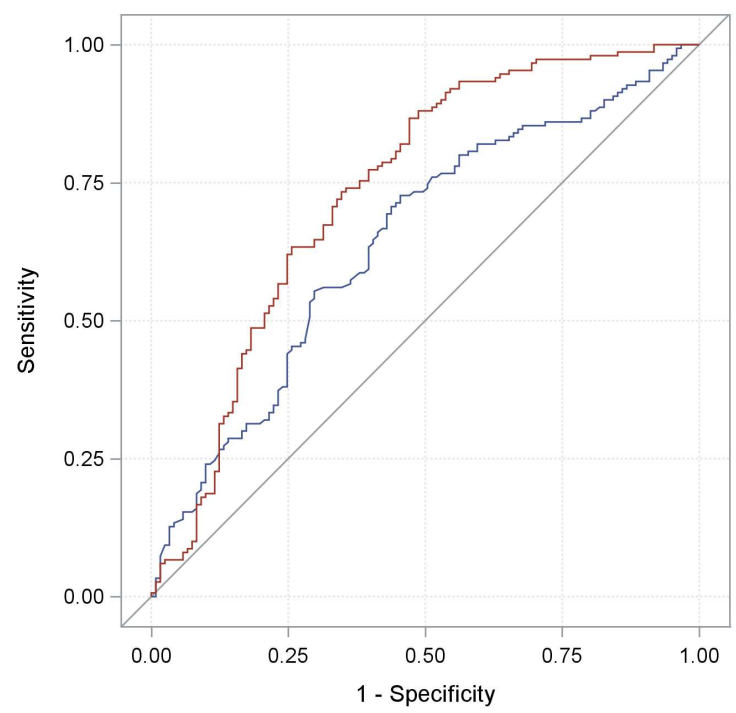

Figure 4. ROC curves of the proposed predictor (red line, AUC: 0.737 ) and N/L ratio one (blue line, AUC: 0.645).

\section{Discussion}

The renewed interest for immunotherapy in the last years and the recent introduction of several ICIs in the clinical practice have redefined the therapeutic strategies of different solid tumors. The efficacy of the immunological approach was first proven in advanced melanoma with the anti CTLA-4 mAb Ipilimumab [20]. Thereafter, also anti PD-1/PD-L1 mAbs were tested against tumors that were classically considered to be poorly immunogenic and mostly unresponsive to immunotherapy, such as non-small cell lung cancer (NSCLC); however, these drugs demonstrated impressive and long-lasting anticancer activity in a minority of patients [21-25]. Unfortunately, despite the remarkable clinical efficacy and low toxicity of ICIs, the vast majority of patients with advanced solid cancers fail to achieve durable responses with ICIs. Therefore, predictive biomarkers of clinical benefit from ICIs are urgently needed in order to select patients with a higher probability of response, as well as to optimize the available economic resources. PD-L1 expression, tumor microenvironment (TME) features, mutational load, mismatch-repair deficiency, and N/L ratio in peripheral blood have been extensively investigated [26]. However, a universally recognized biomarker is not available, yet. For example, although high intratumor PD-L1 expression seems to be significantly associated with a better response to PD-1/PD-L1 blockade agents in several tumors [27], the spatial heterogeneity and dynamic changes of expression in the same tumor, together with the lack of reliable detection methods and definite cut-off values, actually limits its widespread use in clinical practice.

In contrast, measuring LDH serum level is a simple and low-cost evaluation that has already been proposed as a biomarker predictive of tumor response/resistance to regorafenib [28], temsirolimus [29], sorafenib [30,31], and anti CTLA-4 mAbs [12] in patients with colorectal, renal, pancreatic cancers, HCC, and melanoma, respectively.

LDH serum levels have been historically considered to reflect the total number of viable and biologically active cancer cells inside a tumor mass; therefore, its inverse association with patient prognosis and/or tumor response to chemotherapy was mainly attributed to the association between serum LDH levels and tumor burden. However, LDH is a metabolic enzyme that takes crucially part to the glycolytic pathway, which is aberrantly activated in several human cancers to fuel tumor bioenergetics and anabolic needs [32,33]. Of note, enhanced glycolysis in cancer masses leads to reduced glucose levels in the TME and, consequently, to glucose starvation in cells of the TME, including cytotoxic lymphocytes that mediate the antitumor immune response. This metabolic competition between cancer cells and immune cells for the use of glucose molecules may be one crucial mechanism through which malignant cells inhibit the activity of cytotoxic lymphocytes. 
Therefore, the observed inverse association between serum LDH levels and clinical benefit from ICIs could reflect an impairment of antitumor immunity in highly glycolytic, LDH-overexpressing malignancies. An alternative explanation for the link between tumor glycolytic activity and response to immunotherapy comes from a recently published preclinical study, where the inhibition of glycolysis in different tumor models was associated with reduced secretion of Granulocyte Colony-Stimulating Factor (G-CSF) and Granulocyte-Macrophage Colony-Stimulating Factor (GM-CSF) by cancer cells and lower intratumor infiltration by MDSCs, which restrain the activity of cytotoxic lymphocytes [15]. Therefore, high serum LDH, which reflects tumor glycolytic activity, may also reflect a more immunosuppressive, MDSC-enriched tumor microenvironment.

In order to improve the predictive capability of the LDH serum level, we combined it with other clinical parameters for which a rationale exists to test them as predictive biomarkers. To this aim, we have created an interactive Excel tool based on three variables (baseline LDH, age and PS ECOG score) which is able to provide high accuracy in response prediction to ICIs if compared with LDH alone. Our retrospective analysis confirms that patients with high baseline LDH serum levels have a statistically significant reduced probability of achieving a clinical response during treatment with ICIs, especially in patients who are younger and have poorer performance status.

Since the N/L ratio, which reflects systemic cancer-related inflammatory status, has been proposed as a biomarker of resistance to chemo-immunotherapy, we decided to evaluate its impact on the patient's response to ICIs [34]. We observed that the N/L ratio has a significant predictive role but only in univariate fashion and shows a worse predictive capability than our model. To assess the performance of our algorithm, we tried to test it in three of the four colorectal cancer tissues of whom microsatellite instability (MSI) has been previously evaluated: two out of the three patients scored MSI. It is well known that the MSI subset of colorectal cancer has a greater likelihood of response to ICIs compared to the stable one [35]. It is worth noting that our predictor was able to detect with high accuracy which one between the two MSI patients had a major probability of DC and can really benefit from ICIs (Table 4).

Table 4. Estimated probability of response in colorectal patients according to microsatellite mutational status.

\begin{tabular}{cccccc}
\hline PS (ECOG) & Baseline LDH & Age (years) & $\begin{array}{c}\text { Estimated Probability of } \\
\text { Response (\%) }\end{array}$ & Best Response & $\begin{array}{c}\text { Microsatellite } \\
\text { Instability }\end{array}$ \\
\hline 0 & 1063 & 60 & 8.21 & DP & Yes \\
\hline 0 & 354 & 67 & 69.45 & SD & Yes \\
\hline 1 & 925 & 47 & 5.29 & DP & No \\
\hline
\end{tabular}

PS: performance status, DP: disease progression, SD: stable disease.

The algorithm that we developed and validated in this study could provide a base to guide physicians in a real-life setting to better plan a therapeutic strategy tailored to patient characteristics and potentially able to identify patients more likely to benefit from ICIs. The clinical relevance of our findings is related to the easy detectability and manageability of the variables tested. Indeed, information about age, baseline LDH serum levels and PS can be collected quickly, already during the medical examination.

The main limits of our study consist in its retrospective nature, the heterogeneity of tumor histologies included, and the relatively small number of patients enrolled. Prospective studies in larger populations and focused on specific tumor types have already started in order to validate our results. 


\section{Materials and Methods}

\subsection{Ldh Evaluation}

The evaluation of LDH was performed at baseline. All measures were performed in our laboratory, with COBAS ${ }^{\circledR} 6000$ analyser (Roche Diagnostics, Indianapolis, IN, USA), using a UV-test. The catalytic activity of LDH was determined by the measurement of decreased absorbancy of nicotinamide adenine dinucleotide at $340 \mathrm{~nm}$ as a result of catalytic reduction of pyruvate to lactate. From January 2013 to July 2015, the normality reference range was 230-460 Units per liter (U/L); since August 2015, it has changed to $20-480 \mathrm{U} / \mathrm{L}$.

\subsection{Statistical Analysis}

Discrete variables (line and type of therapy (anti-PD1 vs. anti-PDL1), number of metastatic sites, histology, and sex) were opportunely categorized by taking into consideration their clinical function and according to their distributions. Concerning the continuous variables, age and N/L ratio were used in their original scale, whereas for $\mathrm{LDH}$, an appropriate transformation was applied to the original values in order to normalize levels determined by the UV-test with the two different normal reference ranges. All the analyses were performed in terms of percentage increase with respect to the upper limit of each specific range ( $L D H$ normalized). In order to investigate the relationship between the clinical response and continuous variables and to detect possible nonlinear effects, we resorted to a logistic regression model based on restricted cubic splines. A training set, consisting of all patients treated before the 1 st of January 2016 ( $\approx 70 \%$ of all patients), was used to build models which were tested on a validation set including patients treated since the 1 st of January $2016(\approx 30 \%$ of all patients). The relationships between each variable and the clinical response (DC vs. DP) were investigated by resorting to a logistic regression model in both univariate and multivariate fashion. The hypothesis of OR equal to 1 was tested using the Wald Statistic. All the variables resulted statistically significant $(\alpha=0.05)$ in univariate analysis was considered in the initial model of multivariate analysis, and a backward selection procedure was used to obtain the final model. We investigated the predictive capability of the multivariate model by means of the AUC. The nonparametric approach of DeLong and Clarke-Pearson [36] was used to compare the discriminatory performance of different models and evaluate the contribution of each variable of the model. The most satisfactory model was applied on the validation set to statistically validate it and it was fitted overall to obtain the most robust estimates. AUC estimates based on cross-validated predicted probabilities were determined to evaluate the performance of the selected variables in the absence of an independent dataset [37]. All statistical analyses were carried out with SAS software (Version 9.4.; SAS Institute, Inc., Cary, NC, USA) by adopting a significance level of $\alpha=0.05$. The overall coefficients estimated were used to implement an Excel algorithm that requires the selected clinical variables of the patient and returns the corresponding response probability.

\section{Conclusions}

Identifying responder patients before starting immunotherapy is an important clinical need in order to define the best therapeutic workup, avoid unnecessary side effects and efficiently use the economic resources. The clinical relevance of our findings is related to the easy detectability and manageability of the variables tested. Indeed, information about age, baseline LDH serum levels and PS can be collected quickly, already during the medical examination.

The main limits of our study consist in its retrospective nature, the heterogeneity of tumor histologies included, and the relatively small number of patients enrolled. Prospective studies in larger populations and focused on specific tumor types have already started in order to validate our results.

Author Contributions: M.S.C.: conceptualization, data curation, methodology, writing-original draft, writing-review and editing. M.L.: formal analysis, data curation, software, methodology, writing-original draft, writing-review and editing. S.C.: data curation, writing-review and editing. S.D.: data curation, writing-review 
and editing. M.D.V.: data curation, writing-review and editing. A.N.: data curation, writing-review and editing. M.M.P.: data curation. D.R.: data curation, writing-review and editing. G.R.: data curation, writing-review and editing. R.R.: data curation, writing-review and editing. D.S.: data curation, writing-review and editing. C.V.: data curation, writing-review and editing. F.d.B.: conceptualization, methodology, supervision, writing-review and editing. P.V.: formal analysis, data curation, software, methodology, writing-original draft, writing-review and editing. M.D.N.: conceptualization, methodology, supervision, writing-review and editing.

Funding: This research received no external funding.

Conflicts of Interest: The authors declare no conflict of interest.

\section{References}

1. Drent, M.; Cobben, N.A.; Henderson, R.F.; Wouters, E.F.; van Dieijen-Visser, M. Usefulness of lactate dehydrogenase and its isoenzymes as indicators of lung damage or inflammation. Eur. Respir J. 1996, 9 , 1736-1742. [CrossRef] [PubMed]

2. Kemp, M.; Donovan, J.; Higham, H.; Hooper, J. Biochemical markers of myocardial injury. Br. J. Anaesth. 2004, 93, 63-73. [CrossRef] [PubMed]

3. Kato, G.J.; McGowan, V.; Machado, R.F.; Little, J.A.; Taylor, V.I.J.; Morris, C.R.; Nichols, J.S.; Wang, X.; Poljakovic, M.; Morris, S.M.; et al. Lactate dehydrogenase as a biomarker of hemolysis-associated nitric oxide resistance, priapism, leg ulceration, pulmonary hypertension, and death in patients with sickle cell disease. Blood 2006, 107, 2279-2285. [CrossRef] [PubMed]

4. Vander Heiden, M.G.; Cantley, L.C.; Thompson, C.B. Understanding the Warburg Effect: The Metabolic Requirements of Cell Proliferation. Science 2009, 324, 1029-1033. [CrossRef] [PubMed]

5. Motzer, R.J.; Escudier, B.; Bukowski, R.; Rini, B.I.; Hutson, T.E.; Barrios, C.H.; Lin, X.; Fly, K.; Matczak, E.; Gore, M.E. Prognostic factors for survival in 1059 patients treated with sunitinib for metastatic renal cell carcinoma. Br. J. Cancer 2013, 108, 2470-2477. [CrossRef] [PubMed]

6. Wan, X.B.; Wei, L.; Li, H.; Dong, M.; Lin, Q.; Ma, X.K.; Huang, P.Y.; Wen, J.Y.; Li, X.; Chen, J.; et al. High pretreatment serum lactate dehydrogenase level correlates with disease relapse and predicts an inferior outcome in locally advanced nasopharyngeal carcinoma. Eur. J. Cancer 2013, 49, 2356-2364. [CrossRef]

7. Hagberg, H.; Siegbahn, A. Prognostic level of serum lactic dehydrogenase in non-Hodgkin's lymphoma. Scand J. Haematol. 1983, 31, 49-56. [CrossRef]

8. Simonsson, B.; Grenning, G.; Källander, C.; Ahre, A. Prognostic level of serum lactic dehydrogenase (S-LDH) in multiple myeloma. Eur. J. Clin. Investig. 1987, 17, 336-349. [CrossRef]

9. Brereton, H.D.; Simon, R.; Pomeroy, T.C. Pretreatment lactate dehydrogenase predicting metastatic spread in Ewing's sarcoma. Ann. Intern. Med. 1975, 83, 352-364. [CrossRef]

10. Hermes, A.; Gatzemeier, U.; Waschki, B.; Reck, M. Lactate dehydrogenase as prognostic factor in limited and extensive disease stage small cell lung cancer e A retrospective single institution analysis. Respir. Med. 2010, 104, 1937-1942. [CrossRef]

11. Giroux Leprieur, E.; Lavole, A.; Ruppert, A.M.; Gounant, V.; Wislez, M.; Cadranel, J.; Milleron, B. Factors associated with long-term survival of patients with advanced non-small cell lung cancer. Respirology 2012, 17, 134-142. [CrossRef] [PubMed]

12. Diem, S.; Kasenda, B.; Spain, L.; Martin-Liberal, J.; Marconcini, R.; Gore, M.; Larkin, J. Serum lactate dehydrogenase as an early marker for outcome in patients treated with anti-PD-1 therapy in metastatic melanoma. Br. J. Cancer 2016, 114, 256-261. [CrossRef] [PubMed]

13. Kelderman, S.; Heemskerk, B.; van Tinteren, H.; van den Brom, R.R.; Hospers, G.A.; van den Eertwegh, A.J.; Kapiteijn, E.W.; de Groot, J.W.; Soetekouw, P.; Jansen, R.L.; et al. Lactate dehydrogenase as a selection criterion for ipilimumab treatment in metastatic melanoma. Cancer Immunol. Immunother. 2014, 63, 449-458. [CrossRef] [PubMed]

14. Manola, J.; Atkins, M.; Ibrahim, J.; Kirkwood, J. Prognostic factors in metastatic melanoma: A pooled analysis of Eastern Cooperative Oncology Group trials. J. Clin. Oncol. 2000, 18, 3782-3793. [CrossRef] [PubMed]

15. Li, W.; Tanikawa, T.; Kryczek, I.; Xia, H.; Li, G.; Wu, K.; Wei, S.; Zhao, L.; Vatan, L.; Wen, B. Aerobic Glycolysis Controls Myeloid-Derived Suppressor Cells and Tumor Immunity via a Specific CEBPB Isoform in Triple-Negative Breast Cancer. Cell Metab. 2018, 28, 87-103. [CrossRef] [PubMed]

16. Meng, X.; Huang, Z.; Teng, F.; Xing, L.; Yu, J. Predictive biomarkers in PD-1/PD-L1 checkpoint blockade immunotherapy. Cancer Treat Rev. 2015, 41, 868-876. [CrossRef] [PubMed] 
17. Oken, M.M.; Creech, R.H.; Tormey, D.C.; Horton, J.; Davis, T.E.; McFadden, E.T.; Carbone, P.P. Toxicity and response criteria of the Eastern Cooperative Oncology Group. Am. J. Clin. Oncol. 1982, 5, 649-655. [CrossRef]

18. Eisenhauer, E.A.; Therasse, P.; Bogaerts, J.; Schwartz, L.H.; Sargent, D.; Ford, R.; Dancey, J.; Arbuck, S.; Gwyther, S.; Mooney, M.; et al. New response evaluation criteria in solid tumours: Revised RECIST guideline (version 1.1). Eur. J. Cancer 2009, 45, 228-247. [CrossRef]

19. Salvianti, F.; Pinzani, P.; Verderio, P.; Ciniselli, C.M.; Massi, D.; De Giorgi, V.; Grazzini, M.; Pazzagli, M.; Orlando, C. Multiparametric analysis of cell-free DNA in melanoma patients. PLoS ONE 2012, 7, e49843. [CrossRef]

20. Hodi, F.S.; O’Day, S.J.; McDermott, D.F.; Weber, R.W.; Sosman, J.A.; Haanen, J.B.; Gonzalez, R.; Robert, C.; Schadendorf, D.; Hassel, J.C.; et al. Improved survival with ipilimumab in patients with metastatic melanoma. N. Engl. J. Med. 2010, 363, 711-723. [CrossRef]

21. Garon, E.B.; Rizvi, N.A.; Hui, R.; Leighl, N.; Balmanoukian, A.S.; Eder, J.P.; Patnaik, A.; Aggarwal, C.; Gubens, M.; Horn, L.; et al. Pembrolizumab for the treatment of non-small-cell lung cancer. N. Engl. J. Med. 2015, 372, 2018-2028. [CrossRef] [PubMed]

22. Brahmer, J.; Reckamp, K.L.; Baas, P.; Crinò, L.; Eberhardt, W.E.; Poddubskaya, E.; Antonia, S.; Pluzanski, A.; Vokes, E.E.; Holgado, E.; et al. Nivolumab versus docetaxel in advanced squamous-cell non-small-cell lung cancer. N. Engl. J. Med. 2015, 373, 123-135. [CrossRef] [PubMed]

23. Borghaei, H.; Paz-Ares, L.; Horn, L.; Spigel, D.R.; Steins, M.; Ready, N.E.; Chow, L.Q.; Vokes, E.E.; Felip, E.; Holgado, E.; et al. Nivolumab versus docetaxel in advanced nonsquamous non-small-cell lung cancer. N. Engl. J. Med. 2015, 373, 1627-1639. [CrossRef]

24. Motzer, R.J.; Escudier, B.; McDermott, D.F.; George, S.; Hammers, H.J.; Srinivas, S.; Tykodi, S.S.; Sosman, J.A.; Procopio, G.; Plimack, E.R.; et al. Nivolumab versus everolimus in advanced renal-cell carcinoma. N. Engl. J. Med. 2015, 373, 1803-1813. [CrossRef]

25. Balar, A.V.; Galsky, M.D.; Rosenberg, J.E.; Powles, T.; Petrylak, D.P.; Bellmunt, J.; Loriot, Y.; Necchi, A.; Hoffman-Censits, J.; Perez-Gracia, J.L.; et al. Atezolizumab as first-line treatment in cisplatin-ineligible patients with locally advanced and metastatic urothelial carcinoma: A single-arm, multicentre, phase 2 trial. Lancet 2017, 389, 67-76. [CrossRef]

26. Taube, J.M.; Klein, A.; Brahmer, J.R.; Xu, H.; Pan, X.; Kim, J.H.; Chen, L.; Pardoll, D.M.; Topalian, S.L.; Anders, R.A. Association of PD-1, PD-1 ligands, and other features of the tumor immune microenvironment with response to anti-PD-1 therapy. Clin. Cancer Res. 2014, 20, 5064-5074. [CrossRef] [PubMed]

27. Daud, A.I.; Wolchok, J.D.; Robert, C.; Hwu, W.J.; Weber, J.S.; Ribas, A.; Hodi, F.S.; Joshua, A.M.; Kefford, R.; Hersey, P.; et al. Programmed Death-Ligand 1 Expression and Response to the Anti-Programmed Death 1 Antibody Pembrolizumab in Melanoma. J. Clin. Oncol. 2016, 34, 4102-4109. [CrossRef] [PubMed]

28. Del Prete, M.; Giampieri, R.; Loupakis, F.; Prochilo, T.; Salvatore, L.; Faloppi, L.; Bianconi, M.; Bittoni, A.; Aprile, G.; Zaniboni, A.; et al. Prognostic clinical factors in pretreated colorectal cancer patients receiving regorafenib: Implications for clinical management. Oncotarget 2015, 6, 33982-33992. [CrossRef]

29. Armstrong, A.J.; George, D.J.; Halabi, S. Serum lactate dehydrogenase predicts for overall survival benefit in patients with metastatic renal cell carcinoma treated with inhibition of mammalian target of rapamycin. J. Clin. Oncol. 2012, 30, 3402-3417. [CrossRef]

30. Faloppi, L.; Bianconi, M.; Giampieri, R.; Sobrero, A.; Labianca, R.; Ferrari, D.; Barni, S.; Aitini, E.; Zaniboni, A.; Boni, C.; et al. The value of lactate dehydrogenase serum levels as a prognostic and predictive factor for advanced pancreatic cancer patients receiving sorafenib. Oncotarget 2015, 6, 35087-35094. [CrossRef]

31. Faloppi, L.; Scartozzi, M.; Bianconi, M.; Svegliati Baroni, G.; Toniutto, P.; Giampieri, R.; Del Prete, M.; De Minicis, S.; Bitetto, D.; Loretelli, M.; et al. The role of LDH serum levels in predicting global outcome in HCC patients treated with sorafenib: implications for clinical management. PLoS ONE 2012, 7, e32653. [CrossRef]

32. Martinez-Outschoorn, U.E.; Peiris-Pagés, M.; Pestell, R.G.; Sotgia, F.; Lisanti, M.P. Cancer metabolism: a therapeutic perspective. Nat. Rev. Clin. Oncol. 2017, 14, 11-31. [CrossRef] [PubMed]

33. Vernieri, C.; Casola, S.; Foiani, M.; Pietrantonio, F.; de Braud, F.; Longo, V. Targeting Cancer Metabolism: Dietary and Pharmacologic Interventions. Cancer Discov. 2016, 6, 1315-1333. [CrossRef] [PubMed]

34. Mei, Z.; Shi, L.; Wang, B.; Yang, J.; Xiao, Z.; Du, P.; Wang, Q.; Yang, W. Prognostic role of pretreatment blood neutrophil-to-lymphocyte ratio in advanced cancer survivors: A systematic review and meta-analysis of 66 cohort studies. Cancer Treat. Rev. 2017, 58, 1-13. [CrossRef] [PubMed] 
35. Xiao, Y.; Freeman, G.J. The microsatellite instable subset of colorectal cancer is a particularly good candidate for checkpoint blockade immunotherapy. Cancer Discov. 2015, 5, 16-28. [CrossRef]

36. DeLong, E.R.; DeLong, D.M.; Clarke-Pearson, D.L. Comparing the areas under two or more correlated receiver operating characteristic curves: a nonparametric approach. Biometrics 1988, 44, 837-845. [CrossRef] [PubMed]

37. Wu, B.; Abbott, T.; Fishman, D.; McMurray, W.; Mor, G.; Stone, K.; Ward, D.; Williams, K.; Zhao, H. Comparison of statistical methods for classification of ovarian cancer using mass spectrometry data. Bioinformatics 2003, 19, 1636-1643. [CrossRef]

(C) 2019 by the authors. Licensee MDPI, Basel, Switzerland. This article is an open access article distributed under the terms and conditions of the Creative Commons Attribution (CC BY) license (http:/ / creativecommons.org/licenses/by/4.0/). 